\title{
DYNAMICS OF A ONE-PREY AND TWO-PREDATOR SYSTEM WITH TWO HOLLING TYPE FUNCTIONAL RESPONSES AND IMPULSIVE CONTROLS
}

\author{
HUNKI BAEK
}

\author{
Department of Mathematics Education, Catholic University of Daegu, South Korea \\ E-mail address: hkbaek@cu.ac.kr
}

\begin{abstract}
In this paper, we investigate the dynamic behaviors of a one-prey and two-predator system with Holling-type II functional response and defensive ability by introducing a proportion that is periodic impulsive harvesting for all species and a constant periodic releasing, or immigrating, for predators at different fixed time. We establish conditions for the local stability and global asymptotic stability of prey-free periodic solutions by using Floquet theory for the impulsive equation, small amplitude perturbation skills. Also, we prove that the system is uniformly bounded and is permanent under some conditions via comparison techniques. By displaying bifurcation diagrams, we show that the system has complex dynamical aspects.
\end{abstract}

\section{INTRODUCTION}

During the recent years, the impulsive prey-predator population models have been discussed by a number of researchers $[13,14,15,22,27,29]$ and there are also many publications on simple multi-species systems consisting of a three-species food chain with impulsive perturbations $[2,3,8,23,24,26,28]$. A major portion of the literatures involves two aspects: single impulsive control strategy (only biological control or only chemical control) and combination of biological control with chemical control in a pair species. In those papers, the authors assumed that the pest(prey) is portrayed as only being fed on one kind of natural enemy. But, usually the pest has several natural enemies. For examples, aphids in alfalfa have many natural enemies such as convergent lady beetles, big-eyed bugs and Syrphid flies and so on. In this regard, we will establish a one-prey(pest) and two-predator(natural enemies) food web system with biological and chemical controls.

In view of the fact that in population dynamics the reciprocal actions between prey and predator are often complicated and diverse, Holling [10] gave three different kinds of functional responses of the predator to the prey, which refer to the change in the density of the prey attacked per unit time per predator as the prey density changed, which made the standard Lotka-Volterra system more realistic. If we consider the time a predator uses in handling the

Received by the editors August 13 2011; Revised September 10 2012; Accepted in revised form September 15 2012.

2000 Mathematics Subject Classification. 34D23,34A37,34H05.

Key words and phrases. Holling type II, Holling type IV, defensive ability, predator-prey system.

This work was supported by research grants from the Catholic University of Daegu in 2012. 
prey it has captured, one finds the functional response is of Holling type-II, which is monotonic in the first quadrant. However there are experimental and observational evidences that point out that nonmonotonic responses occur because when the nutrient concentration reaches a high level an inhibitory effect on the specific growth rate may occur $[1,25]$. To describe such phenomenon, Andrews [1] suggested a function $p(x)=\frac{m x}{a+b x+x^{2}}$ called the Monod-Haldane function, or Holling type-IV function. Sokol and Howell [19] proposed a simplified Holling type-IV function of the form: $p(x)=\frac{m x}{a+x^{2}}$ which gives an account of the phenomenon of group defense whereby predation is decreased, or even prevented together, due to the increased ability of the prey to better defend or disguise themselves when their numbers are large enough.

The four kinds of Holling-type functional responses have been observed [5, 18]. According to Hassel et al [9], the Holling type II functional response is the most common type of functional responses among arthropod predators. Thus, based on the predator-prey system with Holling II and group defense, we propose an impulsive differential equation to model the process of periodically releasing natural enemies and of spraying pesticides at different fixed times in section 2. Such impulsive systems are found in almost every domain of applied science and have been studied in many investigations: impulsive birth [17, 21], impulsive vaccination $[7,20]$, chemotherapeutic treatment of disease $[12,16]$. In section 4 , we analyze the dynamic behaviors of such systems. Especially, by using Floquet theory for the impulsive equation, small amplitude perturbation skills and comparison techniques, we show that there exists an asymptotically stable pest-free periodic solution and the system is uniformly bounded. In addition, we find a sufficient condition that makes the system permanent. Furthermore, in section 5 , we illustrate several dynamical behaviors including bifurcation diagrams via numerical simulations to give numerical evidences that the system has complex dynamical aspects. Finally, we have a discussion.

Thus, in this paper, we will show the following main results;

Theorem 4.1 The periodic solution $\left(0, y_{1}^{*}(t), y_{2}^{*}(t)\right)$ of system (2.2) is locally asymptotically stable if

$$
a T+\ln \left(1-p_{1}\right)<\sum_{i=1}^{2} c_{i} A_{i}
$$

where $A_{i}=\frac{q_{i}\left(1-\left(1-p_{i+1}\right) \exp \left(-d_{i} T\right)-p_{i+1} \exp \left(-d_{i} \tau T\right)\right)}{d_{i}\left(1-\left(1-p_{i+1}\right) \exp \left(-d_{i} T\right)\right)}(i=1,2)$. Furthermore, the periodic solution $\left(0, y_{1}^{*}(t), y_{2}^{*}(t)\right)$ of system (2.2) is globally asymptotically stable if

$$
a T+\ln \left(1-p_{1}\right)<\frac{b c_{1} A_{1}}{b+e_{1} a}+\frac{b^{2} c_{2} A_{2}}{b^{2}+e_{2} a^{2}}
$$

Theorem 4.2 There is a $M>0$ such that $x(t) \leq M, y_{1}(t) \leq M$ and $y_{2}(t) \leq M$ for all $t$ large enough, where $\left(x(t), y_{1}(t), y_{2}(t)\right)$ is a solution of system (2.2). 
Theorem 4.3 System (2.2) is permanent if

$$
a T+\ln \left(1-p_{1}\right)>\sum_{i=1}^{2} c_{i} A_{i}
$$

where $A_{i}(i=1,2)$ are the constants defined in Theorem 4.1.

\section{MODEL FORMULATIONS}

Based on the ideas discussed in Section 1, we propose the following system with Holling type II and IV functional responses.

$$
\left\{\begin{array}{l}
x^{\prime}(t)=x(t)\left(a-b x(t)-\frac{c_{1} y_{1}(t)}{1+e_{1} x(t)}-\frac{c_{2} y_{2}(t)}{1+e_{2} x^{2}(t)}\right) \\
y_{1}^{\prime}(t)=y_{1}(t)\left(-d_{1}+\frac{c_{3} x(t)}{1+e_{1} x(t)}\right) \\
y_{2}^{\prime}(t)=y_{2}(t)\left(-d_{2}+\frac{c_{4} x(t)}{1+e_{2} x^{2}(t)}\right)
\end{array}\right.
$$

where $x(t)$ and $y_{i}(t)(i=1,2)$ represent the population density of one prey and two predators at time $t$, respectively, and all parameters are positive constants. The constant $a$ is the intrinsic growth rate of the prey population, $b$ is the coefficient of intra-specific competition, $c_{i}(i=1,2)$ are the per-capita rates of predation of the predators, $d_{i}(i=1,2)$ are the death rates of the predators, $c_{i}(i=3,4)$ are the rates of conversing preys into the predators and $e_{i}(i=1,2)$ are the half-saturation constants.

Remark 2.1. (1) It is easy to know that the equilibrium $(0,0,0)$ of system (2.1) is unstable. (2) The equilibrium $\left(0, y_{1+}, y_{2+}\right)$ of system (2.1) does not exist, where $y_{i+}>0(i=1,2)$. 
In this paper, with the idea of impulsive control, we will study the following impulsive system with Holling type II and IV functional responses

$$
\left\{\begin{array}{l}
x^{\prime}(t)=x(t)\left(a-b x(t)-\frac{c_{1} y_{1}(t)}{1+e_{1} x(t)}-\frac{c_{2} y_{2}(t)}{1+e_{2} x^{2}(t)}\right), \\
y_{1}^{\prime}(t)=y_{1}(t)\left(-d_{1}+\frac{c_{3} x(t)}{1+e_{1} x(t)}\right), \\
y_{2}^{\prime}(t)=y_{2}(t)\left(-d_{2}+\frac{c_{4} x(t)}{1+e_{2} x^{2}(t)}\right), \\
t \neq(n+\tau-1) T, t \neq n T, \\
\Delta x(t)=-p_{1} x(t), \\
\left.\Delta y_{1}(t)=-p_{2} y_{1}(t),\right\} t=(n+\tau-1) T, \\
\Delta y_{2}(t)=-p_{3} y_{2}(t), \\
\Delta x(t)=0, \\
\left.\Delta y_{1}(t)=q_{1},\right\} t=n T, \\
\Delta y_{2}(t)=q_{2}, \\
\left(x\left(0^{+}\right), y_{1}\left(0^{+}\right), y_{2}\left(0^{+}\right)\right)=\left(x_{0}, y_{01}, y_{02}\right)
\end{array}\right\}
$$

where $T$ is the period of spaying pesticides(harvesting) and the impulsive immigration or stock of the predator, respectively. Here, $\Delta w=w\left(t^{+}\right)-w(t)$, where $w=x$ and $y_{i}(i=1,2)$. The three parameters $0 \leq p_{1}, p_{2}, p_{3}<1$ present the fraction of the prey and the predators which die due to the harvesting or pesticides etc and $q_{i}(i=1,2)$ mean the size of immigration or stock of the predator.

\section{PRELIMINARIES}

Let $\mathbb{R}_{+}=[0, \infty)$ and $\mathbb{R}_{+}^{3}=\left\{\mathbf{x}=(x, y, z) \in \mathbb{R}^{3}: x, y, z \geq 0\right\}$. Denote $\mathbb{N}$ the set of all of positive integers, $\mathbb{R}_{+}^{*}=(0, \infty)$ and $f=\left(f_{1}, f_{2}, f_{3}\right)^{T}$ the right hand of the first three equations in (2.2). Let $V: \mathbb{R}_{+} \times \mathbb{R}_{+}^{3} \rightarrow \mathbb{R}_{+}$, then $V$ is said to belong to class $V_{0}$ if

(1) $V$ is continuous on $((n-1) T,(n+\tau-1) T] \times \mathbb{R}_{+}^{3} \cup((n+\tau-1) T, n T] \times \mathbb{R}_{+}^{3}$ and for each $\mathbf{x} \in \mathbb{R}_{+}^{3}, n \in \mathbb{N}, \lim _{(t, \mathbf{y}) \rightarrow\left((n-1) T^{+}, \mathbf{x}\right)} V(t, \mathbf{y})=V\left((n-1) T^{+}, \mathbf{x}\right)$ and $\lim _{(t, \mathbf{y}) \rightarrow\left((n+\tau-1) T^{+}, \mathbf{x}\right)} V(t, \mathbf{y})=V\left((n+\tau-1) T^{+}, \mathbf{x}\right)$ exist;

(2) $V$ is locally Lipschitzian in $\mathbf{x}$.

Definition 3.1. For $V \in V_{0}$, one defines the upper right Dini derivative of $V$ with respect to the impulsive differential system (2.2) at $(t, \mathbf{x}) \in((n-1) T,(n+\tau-1) T] \times \mathbb{R}_{+}^{3} \cup((n+\tau-$ 1) $T, n T] \times \mathbb{R}_{+}^{3}$ by

$$
D^{+} V(t, \mathbf{x})=\limsup _{h \rightarrow 0+} \frac{1}{h}[V(t+h, \mathbf{x}+h f(t, \mathbf{x}))-V(t, \mathbf{x})] .
$$


The smoothness properties of $f$ guarantee the global existence and uniqueness of solutions of system (2.2) [11].

Definition 3.2. System (2.2) is said to be permanent if there exist two positive constants $m$ and $M$ such that every positive solution $\left(x(t), y_{1}(t), y_{2}(t)\right)$ of system (2.2) with $x_{0}, y_{01}, y_{02}>0$ satisfies $m \leq x(t) \leq M$ and $m \leq y_{i}(t) \leq M$ for sufficiently large $t, i=1,2$.

We will use a comparison result of impulsive differential inequalities. Suppose that $g$ : $\mathbb{R}_{+} \times \mathbb{R}_{+} \rightarrow \mathbb{R}$ satisfies the following hypotheses:

(H) $g$ is continuous on $((n-1) T,(n+\tau-1) T] \times \mathbb{R}_{+} \cup((n+\tau-1) T, n T] \times \mathbb{R}_{+}$and the limits $\lim _{(t, y) \rightarrow\left((n+\tau-1) T^{+}, x\right)} g(t, y)=g\left((n+\tau-1) T^{+}, x\right), \lim _{(t, y) \rightarrow\left(n T^{+}, x\right)} g(t, y)=g\left(n T^{+}, x\right)$ exist and are finite for $x \in \mathbb{R}_{+}$and $n \in \mathbb{N}$.

Lemma 3.3. [11] Suppose $V \in V_{0}$ and

$$
\left\{\begin{array}{l}
D^{+} V(t, \mathbf{x}) \leq g(t, V(t, \mathbf{x})), t \neq(n+\tau-1) T, t \neq n T \\
V\left(t, \mathbf{x}\left(t^{+}\right)\right) \leq \psi_{n}^{1}(V(t, \mathbf{x})), t=(n+\tau-1) T \\
V\left(t, \mathbf{x}\left(t^{+}\right)\right) \leq \psi_{n}^{2}(V(t, \mathbf{x})), t=n T
\end{array}\right.
$$

where $g: \mathbb{R}_{+} \times \mathbb{R}_{+} \rightarrow \mathbb{R}$ satisfies $(H)$ and $\psi_{n}^{1}, \psi_{n}^{2}: \mathbb{R}_{+} \rightarrow \mathbb{R}_{+}$are non-decreasing for all $n \in \mathbb{N}$. Let $r(t)$ be the maximal solution for the impulsive Cauchy problem

$$
\left\{\begin{array}{l}
u^{\prime}(t)=g(t, u(t)), t \neq(n+\tau-1) T, t \neq n T \\
u\left(t^{+}\right)=\psi_{n}^{1}(u(t)), t=(n+\tau-1) T \\
u\left(t^{+}\right)=\psi_{n}^{2}(u(t)), t=n T \\
u\left(0^{+}\right)=u_{0} \geq 0
\end{array}\right.
$$

defined on $[0, \infty)$. Then $V\left(0^{+}, \mathbf{x}_{0}\right) \leq u_{0}$ implies that $V(t, \mathbf{x}(t)) \leq r(t), t \geq 0$, where $\mathbf{x}(t)$ is any solution of (3.1).

We now indicate a special case of Lemma 3.3 which provides estimations for the solution of a system of differential inequalities. For this, we let $P C\left(\mathbb{R}_{+}, \mathbb{R}\right)\left(P C^{1}\left(\mathbb{R}_{+}, \mathbb{R}\right)\right)$ denote the class of real piecewise continuous(real piecewise continuously differentiable) functions defined on $\mathbb{R}_{+}$.

Lemma 3.4. [11] Let the function $u(t) \in P C^{1}\left(\mathbb{R}^{+}, \mathbb{R}\right)$ satisfy the inequalities

$$
\left\{\begin{aligned}
u^{\prime}(t) & \leq f(t) u(t)+h(t), t \neq \tau_{k}, t>0 \\
u\left(\tau_{k}^{+}\right) & \leq \alpha_{k} u\left(\tau_{k}\right)+\theta_{k}, k \geq 0 \\
u\left(0^{+}\right) & \leq u_{0}
\end{aligned}\right.
$$


where $f, h \in P C\left(\mathbb{R}_{+}, \mathbb{R}\right)$ and $\alpha_{k} \geq 0, \theta_{k}$ and $u_{0}$ are constants and $\left(\tau_{k}\right)_{k \geq 0}$ is a strictly increasing sequence of positive real numbers. Then, for $t>0$,

$$
\begin{aligned}
u(t) \leq & u_{0}\left(\prod_{0<\tau_{k}<t} \alpha_{k}\right) \exp \left(\int_{0}^{t} f(s) d s\right)+\int_{0}^{t}\left(\prod_{s \leq \tau_{k}<t} \alpha_{k}\right) \exp \left(\int_{s}^{t} f(\gamma) d \gamma\right) h(s) d s \\
& +\sum_{0<\tau_{k}<t}\left(\prod_{\tau_{k}<\tau_{j}<t} \alpha_{j}\right) \exp \left(\int_{\tau_{k}}^{t} f(\gamma) d \gamma\right) \theta_{k} .
\end{aligned}
$$

Similar result can be obtained when all conditions of the inequalities in the Lemmas 3.3 and 3.4 are reversed.

Using Lemma 3.4, it is easy to prove that the solutions of system (2.2) with strictly positive initial value remain strictly positive as follows:

Lemma 3.5. The positive octant $\left(\mathbb{R}_{+}^{*}\right)^{3}$ is an invariant region for system (2.2).

Now, for fixed $i=1,2$, consider the impulsive differential equation as follows:

$$
\left\{\begin{array}{l}
y_{i}^{\prime}(t)=-d_{i} y_{i}(t), t \neq(n+\tau-1) T, t \neq n T \\
\Delta y_{i}(t)=-p_{i+1} y_{i}(t), t=(n+\tau-1) T \\
\Delta y_{i}(t)=q_{i}, t=n T \\
y_{i}\left(0^{+}\right)=y_{0 i}
\end{array}\right.
$$

The system (3.4) is a periodically forced linear system. It is easy to obtain that

$$
y_{i}^{*}(t)=\left\{\begin{array}{l}
\frac{q_{i} \exp \left(-d_{i}(t-(n-1) T)\right)}{1-\left(1-p_{i+1}\right) \exp \left(-d_{i} T\right)},(n-1) T<t \leq(n+\tau-1) T, \\
\frac{q_{i}\left(1-p_{i+1}\right) \exp \left(-d_{i}(t-(n-1) T)\right)}{1-\left(1-p_{i+1}\right) \exp \left(-d_{i} T\right)},(n+\tau-1) T<t \leq n T,
\end{array}\right.
$$

with $y_{i}^{*}\left(0^{+}\right)=y_{i}^{*}\left(n T^{+}\right)=\frac{q_{i}}{1-\left(1-p_{i+1}\right) \exp \left(-d_{i} T\right)}$ and $y_{i}^{*}\left((n+\tau-1) T^{+}\right)=\frac{q_{i}\left(1-p_{i+1}\right) \exp \left(-d_{i} \tau T\right)}{1-\left(1-p_{i+1}\right) \exp \left(-d_{i} T\right)}$ is the positive periodic solution of (3.4), where $i=1,2$. Moreover, we can obtain that

$$
y_{i}(t)=\left\{\begin{array}{l}
\left(1-p_{i+1}\right)^{n-1}\left(y_{i}\left(0^{+}\right)-\frac{q_{i}\left(1-p_{i+1}\right) e^{-T}}{1-\left(1-p_{i+1}\right) \exp \left(-d_{i} T\right)}\right) \exp \left(-d_{i} t\right) \\
+y_{i}^{*}(t),(n-1) T<t \leq(n+\tau-1) T, \\
\left(1-p_{i+1}\right)^{n}\left(y_{i}\left(0^{+}\right)-\frac{q_{i}\left(1-p_{i+1}\right) e^{-T}}{1-\left(1-p_{i+1}\right) \exp \left(-d_{i} T\right)}\right) \exp \left(-d_{i} t\right)+y_{i}^{*}(t), \\
(n+\tau-1) T<t \leq n T
\end{array}\right.
$$

is a solution of (3.4), where $i=1,2$. From (3.5) and (3.6), we get easily the following result.

Lemma 3.6. Fix $i=1,2$. Then, for every solution $y_{i}(t)$ and every positive periodic solution $y_{i}^{*}(t)$ of system (3.4), it follows that $y_{i}(t)$ tend to $y_{i}^{*}(t)$ as $t \rightarrow \infty$. Thus, the complete expression for the prey-free periodic solution of system (2.2) is obtained $\left(0, y_{1}^{*}(t), y_{2}^{*}(t)\right)$. 


\section{Mathematical AnAlysis on System (2.2)}

In this section, we will prove that the stability of the lowest-level prey free periodic solution $\left(0, y_{1}^{*}(t), y_{2}^{*}(t)\right)$ is globally asymptotically stable under a condition and then will give a sufficient condition for the permanence of the system. In addition, we will show that the system is uniformly bounded.

Theorem 4.1. The periodic solution $\left(0, y_{1}^{*}(t), y_{2}^{*}(t)\right)$ of system (2.2) is locally asymptotically stable if

$$
a T+\ln \left(1-p_{1}\right)<\sum_{i=1}^{2} c_{i} A_{i}
$$

where $A_{i}=\frac{q_{i}\left(1-\left(1-p_{i+1}\right) \exp \left(-d_{i} T\right)-p_{i+1} \exp \left(-d_{i} \tau T\right)\right)}{d_{i}\left(1-\left(1-p_{i+1}\right) \exp \left(-d_{i} T\right)\right)}(i=1,2)$. Furthermore, the periodic solution $\left(0, y_{1}^{*}(t), y_{2}^{*}(t)\right)$ of system (2.2) is globally asymptotically stable if

$$
a T+\ln \left(1-p_{1}\right)<\frac{b c_{1} A_{1}}{b+e_{1} a}+\frac{b^{2} c_{2} A_{2}}{b^{2}+e_{2} a^{2}} .
$$

Proof. First, we show the local stability of the solution $\left(0, y_{1}^{*}(t), y_{2}^{*}(t)\right)$. The local stability of the pest-free periodic solution $\left(0, y_{1}^{*}(t), y_{2}^{*}(t)\right)$ of system (2.2) may be determined by considering the behavior of small amplitude perturbations of the solution. Let $\left(x(t), y_{1}(t), y_{2}(t)\right)$ be any solution of system (2.2). Define $u(t)=x(t), v(t)=y_{1}(t)-y_{1}^{*}(t), w(t)=y_{2}(t)-y_{2}^{*}(t)$. Then they may be written as

$$
\left(\begin{array}{c}
u(t) \\
v(t) \\
w(t)
\end{array}\right)=\Psi(t)\left(\begin{array}{c}
u(0) \\
v(0) \\
w(0)
\end{array}\right)
$$

where $\Psi(t)$ satisfies

$$
\frac{d \Psi}{d t}=\left(\begin{array}{ccc}
a-c_{1} y_{1}^{*}(t)-c_{2} y_{2}^{*}(t) & 0 & 0 \\
c_{3} y_{1}^{*}(t) & -d_{1} & 0 \\
c_{4} y_{2}^{*}(t) & 0 & -d_{2}
\end{array}\right) \Psi(t)
$$

and $\Psi(0)=I$, the identity matrix. So the fundamental solution matrix is

$$
\Psi(t)=\left(\begin{array}{ccc}
\exp \left(\int_{0}^{t} a-c_{1} y_{1}^{*}(s)-c_{2} y_{2}^{*}(s) d s\right) & 0 & 0 \\
\exp \left(\int_{0}^{t} c_{3} y_{1}^{*}(s) d s\right. & \exp \left(-d_{1} t\right) & 0 \\
\exp \left(\int_{0}^{t} c_{4} y_{2}^{*}(s) d s\right. & 0 & \exp \left(-d_{2} t\right)
\end{array}\right) .
$$

The resetting impulsive conditions of system (2.2) become

$$
\left(\begin{array}{l}
u\left((n+\tau-1) T^{+}\right) \\
v\left((n+\tau-1) T^{+}\right) \\
u\left((n+\tau-1) T^{+}\right)
\end{array}\right)=\left(\begin{array}{ccc}
1-p_{1} & 0 & 0 \\
0 & 1-p_{2} & 0 \\
0 & 0 & 1-p_{3}
\end{array}\right)\left(\begin{array}{c}
u((n+\tau-1) T) \\
v((n+\tau-1) T) \\
w((n+\tau-1) T)
\end{array}\right)
$$


and

$$
\left(\begin{array}{c}
u\left(n T^{+}\right) \\
v\left(n T^{+}\right) \\
w\left(n T^{+}\right)
\end{array}\right)=\left(\begin{array}{lll}
1 & 0 & 0 \\
0 & 1 & 0 \\
0 & 0 & 1
\end{array}\right)\left(\begin{array}{c}
u(n T) \\
v(n T) \\
w(n T)
\end{array}\right) .
$$

Note that all eigenvalues of

$$
S=\left(\begin{array}{ccc}
1-p_{1} & 0 & 0 \\
0 & 1-p_{2} & 0 \\
0 & 0 & 1-p_{3}
\end{array}\right) \Psi(T)
$$

are $\lambda_{1}=\left(1-p_{1}\right) \exp \left(\int_{0}^{T}\left(a-c_{1} y_{1}^{*}(t)-c_{2} y_{2}^{*}(t)\right) d t\right), \lambda_{2}=\left(1-p_{2}\right) \exp \left(-d_{1} T\right)<1$ and $\lambda_{3}=\left(1-p_{3}\right) \exp \left(-d_{2} T\right)<1$. Note that, for $i=1,2$,

$$
\int_{0}^{T} y_{i}^{*}(t) d t=\frac{q_{i}\left(1-\left(1-p_{i+1}\right) \exp \left(-d_{i} T\right)-p_{i+1} \exp \left(-d_{i} \tau T\right)\right)}{d_{i}\left(1-\left(1-p_{i+1}\right) \exp \left(-d_{i} T\right)\right)} .
$$

It follows from (4.1) that $\left|\lambda_{1}\right|<1$. Therefore, from the Floquet theory[4], we obtain $\left(0, y_{1}^{*}(t), y_{2}^{*}(t)\right)$ is locally stable.

It is easy to see that the solution $\left(0, y_{1}^{*}(t), y_{2}^{*}(t)\right)$ is locally stable if the condition (4.2) holds. Now, in order to prove the global stability of the pest-free periodic solution, let $\left(x(t), y_{1}(t), y_{2}(t)\right)$ be a solution of system (2.2). From (4.2) we can select a sufficiently small number $\epsilon_{1}>0$ satisfying

$$
\rho=\left(1-p_{1}\right) \exp \left(a T-\frac{b c_{1}\left(A_{1}-\epsilon_{1} T\right)}{b+e_{1}\left(a+b \epsilon_{1}\right)}-\frac{b^{2} c_{2}\left(A_{2}-\epsilon_{1} T\right)}{b^{2}+e_{2}\left(a+b \epsilon_{1}\right)^{2}}\right)<1 .
$$

It follows from the first equation in (2.2) that $x^{\prime}(t) \leq x(t)(a-b x(t))$ for $t \neq(n+\tau-1) T, t \neq$ $n T$ and $x^{\prime}\left(t^{+}\right)=\left(1-p_{1}\right) x(t) \leq x(t)$ for $t=(n+\tau-1) T$. Now, consider the following impulsive differential equation:

$$
\left\{\begin{array}{l}
f^{\prime}(t)=f(t)(a-b u(t)), t \neq(n+\tau-1) T, t \neq n T \\
\Delta f(t)=0, t=(n+\tau-1) T, t=n T \\
f\left(0^{+}\right)=x_{0}
\end{array}\right.
$$

From Lemma 3.3, we have $x(t) \leq f(t)$. Since $f(t) \rightarrow \frac{a}{b}$ as $t \rightarrow \infty, x(t) \leq \frac{a}{b}+\epsilon$ for any $\epsilon>0$ with $t$ large enough. For simplicity we may assume that $x(t) \leq \frac{a}{b}+\epsilon_{1}$ for all $t>0$. Since $y_{i}^{\prime}(t) \geq-d_{i} y_{i}(t)(i=1,2)$, we can obtain from Lemmas 3.3 and 3.6 that

$$
y_{i}(t) \geq v_{i}(t)>y_{i}^{*}(t)-\epsilon_{1}(i=1,2)
$$

for sufficiently large $t$, where $v_{i}(t)$ is a solution of the following impulsive differential equation:

$$
\left\{\begin{array}{l}
v_{i}^{\prime}(t)=-d_{i} v_{i}(t), t \neq(n+\tau-1) T, t \neq n T, \\
\Delta v_{i}(t)=-p_{i+1} v_{i}(t), t=(n+\tau-1) T, \\
\Delta v_{i}(t)=q_{i}, t=n T \\
v_{i}\left(0^{+}\right)=y_{0 i},
\end{array}\right.
$$


for $i=1,2$. Without loss of generality, we may suppose that (4.5) holds for all $t \geq 0$. From system (2.2), we obtain

$$
\left\{\begin{aligned}
& x^{\prime}(t) \leq x(t)\left(a-\frac{c_{1}\left(y_{1}^{*}(t)-\epsilon_{1}\right)}{1+e_{1}\left(\frac{a}{b}+\epsilon_{1}\right)}-\frac{c_{2}\left(y_{2}^{*}(t)-\epsilon_{1}\right)}{1+e_{2}\left(\frac{a}{b}+\epsilon_{1}\right)^{2}}\right), \\
& t \neq(n+\tau-1) T, t \neq n T, \\
& \Delta x(t) \quad=-p_{1} x(t), t=(n+\tau-1) T, \\
& \Delta x(t) \quad=0, t=n T .
\end{aligned}\right.
$$

Integrating (4.7) on $((n+\tau-1) T,(n+\tau) T]$, we get

$$
\begin{aligned}
x((n+\tau) T) \leq & \left(1-p_{1}\right) x((n+\tau-1) T) \exp \left(\int_{(n+\tau-1) T}^{(n+\tau) T} a-\frac{b c_{1}\left(y_{1}^{*}(t)-\epsilon_{1}\right)}{b+e_{1}\left(a+b \epsilon_{1}\right)}\right. \\
& \left.-\frac{b^{2} c_{2}\left(y_{2}^{*}(t)-\epsilon_{1}\right)}{b^{2}+e_{2}\left(a+b \epsilon_{1}\right)^{2}} d t\right)=x((n+\tau-1) T) \rho
\end{aligned}
$$

and hence $x((n+\tau) T) \leq x(\tau T) \rho^{n}$ which implies that $x((n+\tau) T) \rightarrow 0$ as $n \rightarrow \infty$. Further, we obtain, for $t \in((n+\tau-1) T,(n+\tau) T]$,

$$
\begin{aligned}
x(t) \leq & x((n+\tau-1) T+) \exp \left(\int_{(n+\tau-1) T}^{t} a-\frac{b c_{1}\left(y_{1}^{*}(t)-\epsilon_{1}\right)}{b+e_{1}\left(a+b \epsilon_{1}\right)}\right. \\
& \left.-\frac{b^{2} c_{2}\left(y_{2}^{*}(t)-\epsilon_{1}\right)}{b^{2}+e_{1}\left(a+b \epsilon_{1}\right)^{2}} d t\right) \\
& \leq x((n+\tau-1) T) \exp \left(\left(a+b c_{1} \epsilon_{1}+b^{2} c_{2} \epsilon_{1}\right) T\right)
\end{aligned}
$$

which implies that $x(t) \rightarrow 0$ as $t \rightarrow \infty$. Now, take a sufficiently small number $\epsilon_{2}>0$ satisfying $c_{i+2} \epsilon_{2}<d_{i}(i=1,2)$ to prove that $y_{i}(t) \rightarrow y_{i}^{*}(t)(i=1,2)$ as $t \rightarrow \infty$. Without loss of generality, we may assume that $x(t) \leq \epsilon_{2}$ for all $t \geq 0$. It follows from the second and the third equations in (2.2) that, for $t \neq(n+\tau-1) T$ and $t \neq n T$,

$$
y_{i}^{\prime}(t) \leq y_{i}(t)\left(-d_{i}+c_{i+2} \epsilon_{2}\right)(i=1,2) .
$$

Thus, by Lemmas 3.3 and 3.6, we induce that $y_{i}(t) \leq \tilde{y}_{i}{ }^{*}(t)$, where $\tilde{y}_{i}{ }^{*}(t)$ is the periodic solution of (3.4) with $d_{i}$ changed into $d_{i}-c_{i+2} \epsilon_{2}(i=1,2)$. By letting $\epsilon_{1}, \epsilon_{2} \rightarrow 0$, we obtain from (4.5) and (4.8) that $y_{i}(t)$ tends to $y_{i}^{*}(t)$ as $t \rightarrow \infty$.

We show that all solutions of system (2.2) are uniformly bounded.

Theorem 4.2. There is a $M>0$ such that $x(t) \leq M, y_{1}(t) \leq M$ and $y_{2}(t) \leq M$ for all $t$ large enough, where $\left(x(t), y_{1}(t), y_{2}(t)\right)$ is a solution of system (2.2).

Proof. Let $\left(x(t), y_{1}(t), y_{2}(t)\right)$ be a solution of (2.2) with an nonnegative initial condition $\left(x_{0}, y_{01}, y_{02}\right)$ and define $F(t)=x(t)+\frac{c_{1}}{c_{3}} y_{1}(t)+\frac{c_{2}}{c_{4}} y_{2}(t)$ for $t>0$. Then $F^{\prime}(t)+\delta F(t)=-b x^{2}(t)+$ $(a+\delta) x(t)+\frac{c_{1}}{c_{3}}\left(\delta-d_{1}\right) y_{1}(t)+\frac{c_{2}}{c_{4}}\left(\delta-d_{2}\right) y_{2}(t)$ where $t \neq n T$ and $t \neq(n+\tau-1) T$, Select $0<\delta_{0}<\min \left\{d_{1}, d_{2}\right\}$ to show that

$$
F^{\prime}(t)+\delta_{0} F(t) \leq-b x^{2}(t)+\left(a+\delta_{0}\right) x(t)
$$


for $t \neq(n+\tau-1) T, t \neq n T$ and $t>0$. Since $-b x^{2}(t)+\left(a+\delta_{0}\right) x(t)$ is bounded from above by $M_{0}=\frac{\left(a+\delta_{0}\right)^{2}}{b}$, it follows that

$$
F^{\prime}(t)+\delta_{0} F(t) \leq M_{0}, n \neq(n+\tau-1) T, t \neq n T, t>0 .
$$

If $t=n T$, then $F\left(t^{+}\right)=F(t)+q$ and if $t=(n+\tau-1) T$, then $F\left(t^{+}\right) \leq(1-p) F(t)$, where $q=\frac{c_{1}}{c_{3}} q_{1}+\frac{c_{2}}{c_{4}} q_{2}$ and $p=\min \left\{p_{1}, p_{2}, p_{3}\right\}$. From Lemma 3.4, we get that

$$
\begin{aligned}
F(t) \leq & F_{0}(1-p)^{\left[\frac{t}{k T}\right]} \exp \left(\int_{0}^{t}-\delta_{0} d s\right)+\int_{0}^{t}(1-p)^{\left[\frac{t-s}{k T}\right]} \exp \left(\int_{s}^{t}-\delta_{0} d \gamma\right) M_{0} d s \\
& +\sum_{j=1}^{\left[\frac{t}{k T}\right]}(1-p)^{\left[\frac{t-k T}{j T}\right]} \exp \left(\int_{k T}^{t}-\delta_{0} d \gamma\right) q \\
\leq & F_{0} \exp \left(-\delta_{0} t\right)+\frac{M_{0}}{\delta_{0}}\left(1-\exp \left(-\delta_{0} t\right)\right)+\frac{q \exp \left(\delta_{0} T\right)}{\exp \left(\delta_{0} T\right)-1},
\end{aligned}
$$

where $F_{0}=x_{0}+\frac{c_{1}}{c_{3}} y_{01}+\frac{c_{2}}{c_{4}} y_{02}$. Thus, $F(t)$ is bounded by a constant $M \equiv \frac{M_{0}}{\delta_{0}}+\frac{q \exp \left(\delta_{0} T\right)}{\exp \left(\delta_{0} T\right)-1}$ for sufficiently large $t$. Therefore, $x(t), y_{1}(t)$ and $y_{2}(t)$ are bounded for sufficiently large $t$.

Theorem 4.3. System (2.2) is permanent if

$$
a T+\ln \left(1-p_{1}\right)>\sum_{i=1}^{2} c_{i} A_{i},
$$

where $A_{i}(i=1,2)$ are the constants defined in Theorem 4.1.

Proof. Let $\left(x(t), y_{1}(t), y_{2}(t)\right)$ be a solution of system (2.2) with an strictly positive initial condition $\left(x_{0}, y_{01}, y_{02}\right)$. From Theorem 4.2, we may assume that $x(t), y_{1}(t)$ and $y_{2}(t) \leq M$ and $M>c_{1}+c_{2}$. For $\epsilon_{i}>0(i=1,2)$, let $m_{i}=\frac{\left.q_{i}\left(1-p_{i+1}\right) \exp \left(-d_{i} T\right)\right)}{1-\left(1-p_{i+1}\right) \exp \left(-d_{i} T\right)}-\epsilon_{i}$. From Lemmas 3.3 and 3.6 we can obtain that $y_{i}(t)>y_{i}^{*}(t)-\epsilon_{i}(i=1,2)$ for sufficiently large $t$, where $y_{i}^{*}(t)(i=1,2)$ are the periodic solution of (3.4). Hence $y_{i}(t)>m_{i}(i=1,2)$ for sufficiently large $t$. Thus we only need to find $m_{3}$ such that $x(t) \geq m_{3}$ for sufficiently large $t$. We will do this in the following two steps.

Step 1: From (4.11), we can take sufficiently small numbers $m_{3}^{\prime}>0$ and $\epsilon_{i}^{\prime}>0(i=1,2)$ such that $\eta_{i} \equiv d_{i}-c_{i+2} m_{3}^{\prime}>0(i=1,2)$ and

$$
\phi \equiv\left(1-p_{1}\right) \exp \left(\left(a-b m_{3}^{\prime}-c_{1} \epsilon_{1}^{\prime}-c_{2} \epsilon_{2}^{\prime}\right) T-c_{1} A_{\eta_{1}}-c_{2} A_{\eta_{2}}\right)>1,
$$

where $A_{\eta_{i}}=\frac{q_{i}\left(1-\left(1-p_{i+1}\right) \exp \left(-\eta_{i} T\right)-p_{i+1} \exp \left(-\eta_{i} \tau T\right)\right)}{\eta_{i}\left(1-\left(1-p_{i+1}\right) \exp \left(-\eta_{i} T\right)\right)}(i=1,2)$. We will prove there exists $t_{1} \in(0, \infty)$ such that $x\left(t_{1}\right) \geq m_{3}^{\prime}$ by using a contradiction to the boundedness of $x(t)$. Suppose that $x(t)<m_{3}^{\prime}$, for all $t>0$. It follows from system (2.2) that $y_{i}^{\prime}(t) \leq$ $y_{i}(t)\left(-d_{i}+c_{i+2} x(t)\right) \leq y_{i}(t)\left(-d_{i}+c_{i+2} m_{3}^{\prime}\right)=-\eta_{i} y_{i}(t)(i=1,2)$ for $t \neq(n+\tau-1) T, t \neq$ 
$n T$. Thus we get, for $i=1,2, y_{i}(t) \leq v_{i}(t)$ and $v_{i}(t) \rightarrow v_{i}^{*}(t)$ as $t \rightarrow \infty$, where $v_{i}(t)$ is the solution of system

$$
\left\{\begin{array}{l}
v_{i}^{\prime}(t)=-\eta_{i} v_{i}(t), t \neq(n+\tau-1) T, t \neq n T \\
\Delta v_{i}(t)=-p_{i+1} v_{i}(t), t=(n+\tau-1) T \\
\Delta v_{i}(t)=q_{i}, t=n T \\
v_{i}\left(0^{+}\right)=y_{0 i}
\end{array}\right.
$$

and

$$
v_{i}^{*}(t)=\left\{\begin{array}{l}
\frac{q_{i} \exp \left(-\eta_{i}(t-(n-1) T)\right)}{1-\left(1-p_{i+1}\right) \exp \left(-\eta_{i} T\right)},(n-1) T<t \leq(n+\tau-1) T, \\
\frac{q_{i}\left(1-p_{i+1}\right) \exp \left(-\eta_{i}(t-(n-1) T)\right)}{1-\left(1-p_{i+1}\right) \exp \left(-\eta_{i} T\right)},(n+\tau-1) T<t \leq n T .
\end{array}\right.
$$

Therefore, we can take $T_{i}>0$ such that $y_{i}(t) \leq v_{i}(t)<v_{i}^{*}(t)+\epsilon_{i}^{\prime}$ for $t>T_{i}, i=1,2$. Let $T_{1}^{\prime}=\max \left\{T_{1}, T_{2}\right\}$. Thus we get

$$
\begin{cases}x^{\prime}(t) & \geq x(t)\left(a-b m_{3}^{\prime}-\sum_{i=1}^{2} c_{i}\left(v_{i}^{*}(t)+\epsilon_{i}^{\prime}\right)\right), t \neq(n+\tau-1) T, t \neq n T \\ \Delta x(t) & =-p_{1} x(t), t=(n+\tau-1) T \\ \Delta x(t) & =0, t=n T\end{cases}
$$

for $t>T_{1}^{\prime}$. Let $N_{1} \in \mathbb{N}$ be such that $\left(N_{1}+\tau-1\right) T \geq T_{1}^{\prime}$. Integrating the equation (4.15) on $((n+\tau-1) T,(n+\tau) T], n \geq N_{1}$, we can obtain that $x((n+\tau) T) \geq x((n+\tau-$ 1)T $)\left(1-p_{1}\right) \exp \left(\int_{(n+\tau-1) T}^{(n+\tau) T} a-b m_{3}^{\prime}-\sum_{i=1}^{2} c_{i}\left(v_{i}^{*}(t)+\epsilon_{i}^{\prime}\right) d t=x((n+\tau-1) T) \phi\right.$. Thus $x\left(\left(N_{1}+k+\tau\right) T\right) \geq x\left(\left(N_{1}+\tau\right) T\right) \phi^{k} \rightarrow \infty$ as $k \rightarrow \infty$, which is a contradiction .

Step 2: If $x(t) \geq m_{3}^{\prime}$ for all $t \geq t_{1}$, then we are done. If not, we may let $t^{*}=\inf _{t>t_{1}}\{x(t)<$ $\left.m_{3}^{\prime}\right\}$. Then $x(t) \geq m_{3}^{\prime}$ for $t \in\left[t_{1}, t^{*}\right)$ and, by the continuity of $x(t)$, we have $x\left(t^{*}\right)=m_{3}^{\prime}$. In this step, we have only to consider two possible cases.

Case (i): Suppose that $t^{*}=\left(n_{1}+\tau-1\right) T$ for some $n_{1} \in \mathbb{N}$. Then $\left(1-p_{1}\right) m_{3}^{\prime} \leq x\left(t^{*+}\right)=$ $\left(1-p_{1}\right) x\left(t^{*}\right)<m_{3}^{\prime}$. We may assume that $\sigma \equiv a-b m_{3}^{\prime}-\left(c_{1}+c_{2}\right) M<0$ since $M>$ $c_{1}+c_{2}$. Now, select $n_{2 i}, n_{3} \in \mathbb{N}(i=1,2)$ such that $\left(n_{2 i}-1\right) T>-\frac{\ln \left(\frac{\epsilon_{i}^{\prime}}{M+q_{i}}\right)}{\eta_{i}}$ and $(1-$ $\left.p_{1}\right)^{n_{2 i}} \phi^{n_{3}} \exp \left(n_{2 i} \sigma T\right)>\left(1-p_{1}\right)^{n_{2 i}} \phi^{n_{3}} \exp \left(\left(n_{2 i}+1\right) \sigma T\right)>1$. Let $n_{2}=\max \left\{n_{21}, n_{22}\right\}$ and $T^{\prime}=n_{2} T+n_{3} T$. In this case we will show that there exists $t_{2} \in\left(t^{*}, t^{*}+T^{\prime}\right]$ such that $x\left(t_{2}\right) \geq m_{3}^{\prime}$. Otherwise, by (3.6) and (4.13) with $v_{i}\left(n_{1} T^{+}\right)=y_{i}\left(n_{1} T^{+}\right)(i=1,2)$, we have, 
for $i=1,2$,

$$
v_{i}(t)=\left\{\begin{array}{l}
\left(1-p_{i+1}\right)^{n-\left(n_{1}+1\right)}\left(v_{i}\left(n_{1} T^{+}\right)-\frac{q_{i}\left(1-p_{i+1}\right) \exp (-T)}{1-\left(1-p_{i+1}\right) \exp \left(-\eta_{i} T\right)}\right) \\
\exp \left(-\eta_{i}\left(t-n_{1} T\right)\right)+v_{i}^{*}(t),(n-1) T<t \leq(n+\tau-1) T, \\
\left(1-p_{i+1}\right)^{\left(n-n_{1}\right)}\left(v_{i}\left(n_{1} T^{+}\right)-\frac{q_{i}\left(1-p_{i+1}\right) \exp (-T)}{1-\left(1-p_{i+1}\right) \exp \left(-\eta_{i} T\right)}\right) \\
\exp \left(-\eta_{i}\left(t-n_{1} T\right)\right)+v_{i}^{*}(t),(n+\tau-1) T<t \leq n T,
\end{array}\right.
$$

and $n_{1}+1 \leq n \leq n_{1}+1+n_{2}+n_{3}$. So we obtain $\left|v_{i}(t)-v_{i}^{*}(t)\right| \leq\left(M+q_{i}\right) \exp \left(-\eta_{i}(t-\right.$ $\left.\left.n_{1} T\right)\right)<\epsilon_{i}^{\prime}$ and $y_{i}(t) \leq v_{i}(t) \leq v_{i}^{*}(t)+\epsilon_{i}^{\prime}$ for $n_{1} T+\left(n_{2}-1\right) T \leq t \leq t^{*}+T^{\prime}$, which implies (4.15) holds for $t \in\left[t^{*}+n_{2} T, t^{*}+T^{\prime}\right]$. Similarly to step 1, we obtain

$$
x\left(t^{*}+T^{\prime}\right) \geq x\left(t^{*}+n_{2} T\right) \phi^{n_{3}} .
$$

Since $y_{i}(t) \leq M(i=1,2)$, we have

$$
\begin{cases}x^{\prime}(t) & \geq x(t)\left(a-b m_{3}^{\prime}-\left(c_{1}+c_{2}\right) M\right)=\sigma x(t), t \neq n T, t \neq(n+\tau-1) T, \\ \Delta x(t) & =-p_{1} x(t), t=(n+\tau-1) T, \\ \Delta x(t) & =0, t=n T\end{cases}
$$

for $t \in\left[t^{*}, t^{*}+n_{2} T\right]$. Integrating (4.16) on $\left[t^{*}, t^{*}+n_{2} T\right]$ we have

$$
\begin{aligned}
x\left(\left(t^{*}+n_{2} T\right)\right) & \geq m_{3}^{\prime} \exp \left(\sigma n_{2} T\right) \\
& \geq m_{3}^{\prime}\left(1-p_{1}\right)^{n_{2}} \exp \left(\sigma n_{2} T\right) .
\end{aligned}
$$

Thus $x\left(t^{*}+T^{\prime}\right) \geq m_{3}^{\prime}\left(1-p_{1}\right)^{n_{2}} \exp \left(\sigma n_{2} T\right) \phi^{n_{3}}>m_{3}^{\prime}$ which is a contradiction. Now, let $\bar{t}$ $=\inf _{t>t^{*}}\left\{x(t) \geq m_{3}^{\prime}\right\}$. Then $x(t) \leq m_{3}^{\prime}$ for $t^{*} \leq t<\bar{t}$ and $x(\bar{t})=m_{3}^{\prime}$. So, we have, for $t \in\left[t^{*}, \bar{t}\right), x(t) \geq m_{3}^{\prime}\left(1-p_{1}\right)^{n_{2}+n_{3}} \exp \left(\sigma\left(n_{2}+n_{3}\right) T\right) \equiv m_{3}$. For $t>\bar{t}$ the same argument can be continued since $x(\bar{t}) \geq m_{3}^{\prime}\left(>m_{3}\right)$. Hence $x(t) \geq m_{3}$ for all $t>t_{1}$.

Case (ii): $t^{*} \neq(n+\tau-1) T$, for all $n \in \mathbb{N}$. Suppose that $t^{*} \in\left(\left(n_{1}^{\prime}+\tau-1\right) T,\left(n_{1}^{\prime}+\tau\right) T\right)$ for some $n_{1}^{\prime} \in \mathbb{N}$. There are two possible cases for $t \in\left(t^{*},\left(n_{1}^{\prime}+\tau\right) T\right)$. Firstly, if $x(t) \leq m_{3}^{\prime}$ for all $t \in\left(t^{*},\left(n_{1}^{\prime}+\tau\right) T\right)$, similar to case (i), we can prove there must be a $t_{2}^{\prime} \in\left[\left(n_{1}^{\prime}+\right.\right.$ $\left.\tau) T,\left(n_{1}^{\prime}+\tau\right) T+T^{\prime}\right]$ such that $x\left(t_{2}^{\prime}\right) \geq m_{3}^{\prime}$. Here we omit it. Let $\hat{t}=\inf _{t>t^{*}}\left\{x(t) \geq m_{3}^{\prime}\right\}$. Then $x(t) \leq m_{3}^{\prime}$ for $t \in\left(t^{*}, \hat{t}\right]$ and $x(\hat{t})=m_{3}^{\prime}$. For $t \in\left(t^{*}, \hat{t}\right]$, we have $x(t) \geq m_{3}^{\prime}(1-$ $\left.p_{1}\right)^{n_{2}+n_{3}} \exp \left(\sigma\left(n_{2}+n_{3}+1\right) T\right) \equiv m_{3}$. So, $m_{3}<m_{3}^{\prime}$ and $x(t) \geq m_{3}$ for $t \in\left(t^{*}, \hat{t}\right]$. For $t>\hat{t}$ the same argument can be continued since $x(\hat{t}) \geq m_{3}^{\prime}$. Hence $x(t) \geq m_{3}$ for all $t>t_{1}$ Secondly, if there exists a $t \in\left(t^{*},\left(n_{1}^{\prime}+\tau\right) T\right)$ such that $x(t) \geq m_{3}^{\prime}$. Let $\check{t}=$ $\inf _{t>t^{*}}\left\{x(t) \geq m_{3}^{\prime}\right\}$. Then $x(t) \leq m_{3}^{\prime}$ for $t \in\left(t^{*}, \check{t}\right]$ and $x(\check{t})=m_{3}^{\prime}$. For $t \in\left(t^{*}, \check{t}\right]$, we have $x(t) \geq x\left(t^{*}\right) \exp \left(\sigma\left(t-t^{*}\right)\right) \geq m_{3}^{\prime} \exp (\sigma T)>m_{3}^{\prime}>m_{3}$. This process can be continued since $x(\breve{t}) \geq m_{3}^{\prime}$, and have $x(t) \geq m_{3}$ for all $t>t_{1}$. This completes the proof.

Remark 4.4. Define $G(T)=a T+\ln \left(1-p_{1}\right)-\sum_{i=1}^{2} c_{i} A_{i}$. Note that $G(0)=\ln \left(1-p_{1}\right)<0$, $G(T) \rightarrow \infty$ as $T \rightarrow \infty$, and $G^{\prime \prime}(T)>0$. Thus the equation $G(T)=0$ has a unique positive solution, denoted by $T^{*}$. It follows from Theorem 4.1 that the prey-free periodic solution 
$\left(0, y_{1}^{*}(t), y_{2}^{*}(t)\right)$ is locally asymptotically stable if $T<T^{*}$. From Theorem 4.3, we know that if the period of pulse $T$ is larger than $T^{*}$, then the prey-free periodic solution becomes unstable while the system (2.2) becomes permanent. i.e., the prey and the two predators can coexist when $T>T^{*}$. We will exhibit numerical examples in next section to substantiate our theoretical results.

\section{Dynamic VARIETIES OF System (2.2)}

In this section we will illustrate the dynamic varieties of system (2.2) through studying the effects of impulsive period $T$ as a means of control on the system (2.2) by using numerical simulations.

Now, in order to do this, fix the parameters as follows:

$$
\begin{aligned}
& a=6.3, b=1.1, c_{1}=0.6, c_{2}=0.5, c_{3}=0.5, c_{4}=0.6, d_{1}=0.4, \\
& d_{2}=0.3, e_{1}=0.7, e_{2}=0.6 .
\end{aligned}
$$

As known, it is difficult to study systems (2.1) and (2.2) analytically. Thus we numerically integrated the system (2.2) and seek the long-term behavior of the solutions. For this, fix $p_{1}=0.5, p_{2}=0.0001, p_{3}=0.00001, q_{1}=0.1, q_{2}=0.2, \tau=0.6$. As mentioned in Remark 4.4, the prey-free periodic solution is stable when $T<T^{*} \approx 0.236$ (see Figure 1), and the system (2.2) is permanent when $T>T^{*}$ (see Figure 2).

(a)

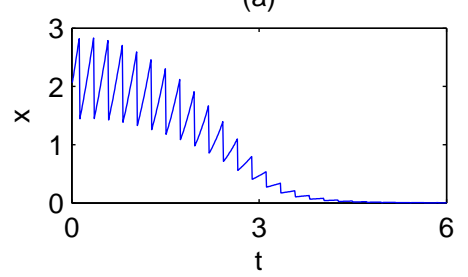

(c)

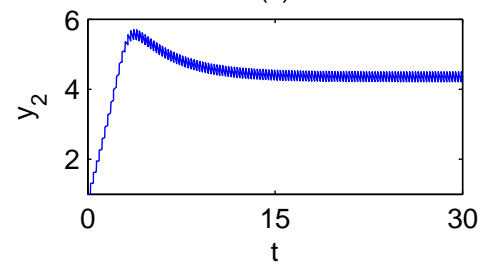

(b)

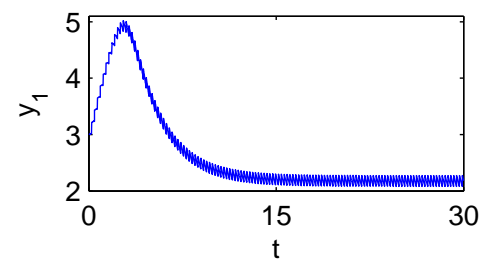

(d)

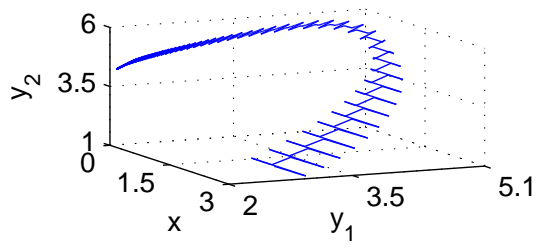

FIGURE 1. Time series of system (2.2) with respect to (a) $x$, (b) $y_{1}$ and (c) $y_{2}$, respectively. (d) The phase portrait of system (2.2) with $\left(x_{0}, y_{10}, y_{20}\right)=$ $(2,3,1)$.

The bifurcation diagrams for the prey and predators populations as $T$ increasing from 0 to 13 with an initial value $\left(x_{0}, y_{01}, y_{02}\right)=(2,3,1)$ are shown in Figure 3 . They illustrate typical dynamical behaviors such as quasi-periodic oscillation(see Figure 4(a)), periodic doubling 
(a)

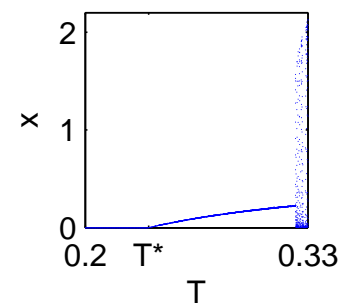

(b)

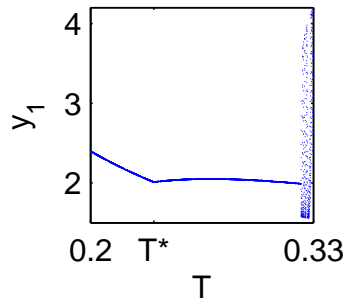

(c)

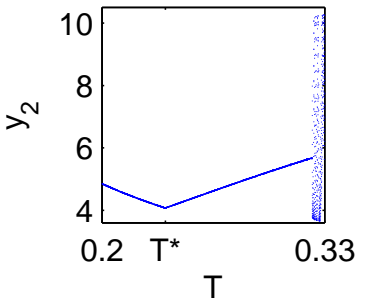

FIGURE 2. Magnitudes of the bifurcation diagrams shown in Figure 3 when $0.2<T<0.33$. Especially, $T^{*}$ indicates the value of $T \approx 0.236$.

(a)

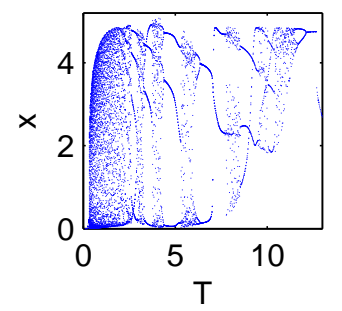

(b)

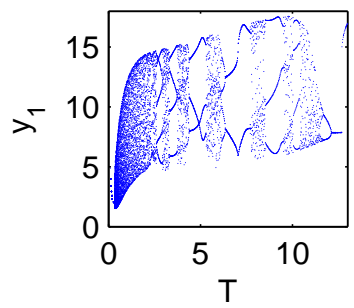

(c)

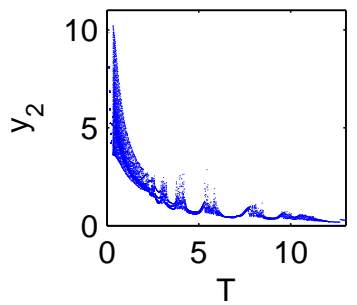

FigURE 3. Bifurcation diagrams of system (2.2) when $\left(x_{0}, y_{0}, z_{0}\right)=$ $(2,3,1)$. (a) $x$, (b) $y_{1}$ and (c) $y_{2}$ are plotted for $T$.

(a)

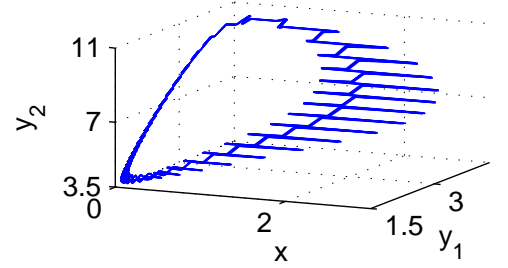

(b)

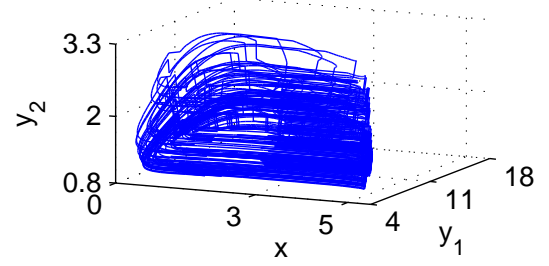

FIGURE 4. Phase portraits of system (2.2) when (a) $T=0.327$ and (b) $T=4$.

(see Figure 5), periodic halving(see Figure 6), periodic windows(see Figure 3), a chaotic area including chaos(see Figure 4(b)) and so on. But there are some interesting phenomena when $T$ varies from 0.3 to 2 . Especially, Figure 7 is shown an enlarged part of the bifurcation diagrams when $0.32<T<0.33$. From these bifurcation diagrams, one could make a mistake to look upon the black regions as a chaotic area. One should notice the banded structure of the quasi-periodic behavior and compare that to the random scatter of the chaotic. For instant, Figure 4(a) displays a high order quasi-periodicity when $T=0.327$, and Figure 4(b) shows a 
(a)

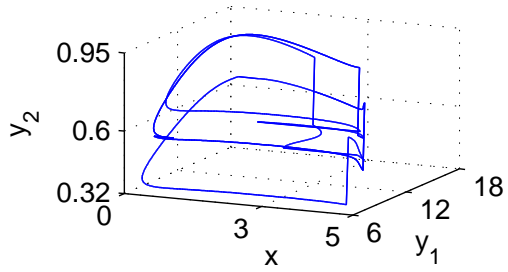

(b)

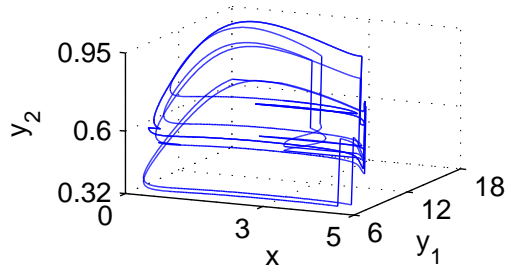

FIgURE 5. Periodic doubling bifurcation of system (2.2). Phase portraits of (a) a $4 T$-period solution for $T=10.39$ and (b) a $8 T$-period solution for $T=$ 10.41 .

(a)

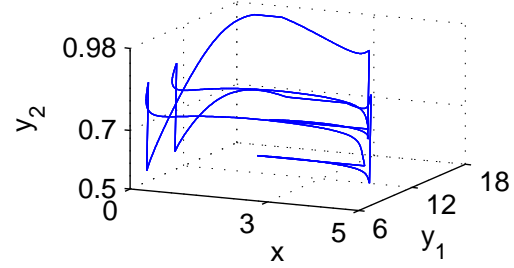

(b)

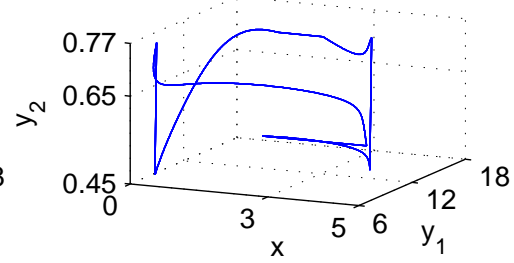

FIGURE 6. Periodic halving bifurcation of system (2.2). Phase portraits of (a) a $4 T$-period solution for $T=6.2$ and (b) a $2 T$-period solution for $T=6.4$.

(a)

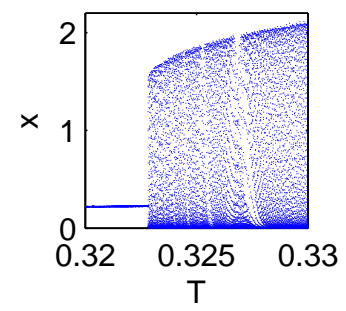

(b)

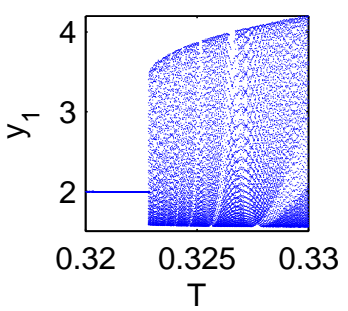

(c)

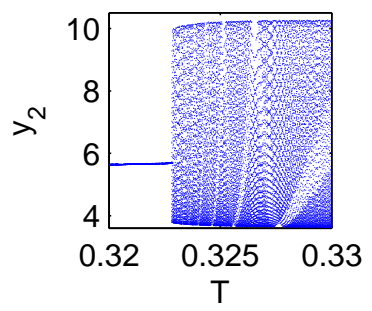

FIGURE 7. Magnitudes of the bifurcation diagrams shown in Figure 3 when $0.32<T<0.33$.

strange attractor when $T=4$. Thus, the dynamic behaviors of the black area include either quasi-periodic aspects or chaotic aspects.

\section{DISCUSSION}

In this paper, we have proposed new impulsive one-prey and two-predator systems with Holling-type II functional response and group defense to model the process of periodically releasing natural enemies and of spraying pesticides at different fixed times. We have shown that there exists a stable prey-free periodic solution when the impulsive period $T$ is less than a 
critical value by using the Floquet theory of impulsive differential equation and small amplitude perturbation skills. When the stabilities of prey-free periodic solutions disappear, the system (2.2) becomes permanent, which is line with reality from a biological point of view. In the following, let us compare the results of system (2.1) with systems (2.2).

System (2.1) is one of models with a classical biological control technique. From Remark 2.1 , it can be shown that the prey-free equilibrium $\left(0, y_{1+}, y_{2+}\right)\left(y_{i+}>0, i=1,2\right)$ of the system (2.1) doesn't exist and the equilibrium $(0,0,0)$ of the system (2.1) is unstable, which indicates that one cannot stamp out the prey(pest) steadily. But, from the impulsive controlled system (2.2), the prey (pest) can be eradicated if the impulsive period $T$ is less than a critical value $T^{*}$. Thus, if one regards the prey as a pest, then the impulsive control strategy is more suitable than the classical one. On the contrary, the impulsive perturbations of the predators should be occurred at long-time intervals for coexistence of the prey and the predators.

\section{REFERENCES}

[1] J. F. Andrews, A mathematical model for the continuous culture of microorganisms utilizing inhibitory substrates, Biotechnol. Bioeng., 10(1968), 707-723.

[2] H. Baek, Dynamic complexites of a three - species Beddington-DeAngelis system with impulsive control strategy, Acta Appl. Math., 110(1)(2010), 23-38.

[3] H. Baek, Qualitative analysis of Beddingtion-DeAngelis type impulsive predator-prey models, Nonlinear Analysis : Real World Applications, 11(3)(2010), 1312-1322.

[4] D.D. Bainov and P.S. Simeonov, Impulsive Differential Equations:asymptotic properties of the solutions, Singapore:World Scientific, 1993.

[5] B. A. Croft, Arthropod biological control agents and pesicides. Wiley, New York (1990).

[6] J. M. Cushing, Periodic time-dependent predator-prey systems, SIAM J. Appl. Math. 32(1977), 82-95.

[7] A. Donofrio, Stability properties of pulse vaccination strategy in SEIR epidemic model, Math. Biosci, 179(2002), 57-72.

[8] P. Georgescu and G. Morosanu, Impulsive perturbations of a three-trophic prey-dependent food chain system, Mathematical and Computer Modeling, 48(2008), 975-997.

[9] M. P. Hassell, The dynamics of competition and predation. p.68. Arnod, London (1976).

[10] C. S. Holling, The functional response of predator to prey density and its role in mimicy and population regulatio. Mem. Entomol. Soc. Can., 45(1965), 1-60.

[11] V. Lakshmikantham, D. Bainov, P.Simeonov, Theory of Impulsive Differential Equations, World Scientific Publisher, Singapore, 1989.

[12] A. Lakmeche and O. Arino, Bifurcation of non trivial periodic solutions of impulsive differential equations arising chemotherapeutic treatment, Dynamics of Continuous, Discrete and Impulsive Systems, 7(2000), 265-287.

[13] B. Liu, Y. Zhang and L. Chen, Dynamic complexities in a Lotka-Volterra predator-prey model concerning impulsive control strategy, Int. J. of Bifur. and Chaos, 15(2)(2005), 517-531.

[14] B. Liu, Z. Teng and L. Chen, Analsis of a predator-prey model with Holling II functional response concerning impulsive control strategy, J. of Comp. and Appl. Math., 193(1)(2006), 347-362

[15] X. Liu and L. Chen, Complex dynamics of Holling type II Lotka-Volterra predator-prey system with impulsive perturbations on the predator, Chaos, Solitons and Fractals, 16(2003), 311-320.

[16] J. C. Panetta, A mathematical model of periodically pulsed chemotherapy: tumor recurrence and metastasis in a competitive environment, Bull. Math. Biol., 58(1996), 425-447.

[17] M. G. Roberts and R. R. Kao, The dynamics of an infectious disease in a population with birth purses, Math. Biosci., 149(1998), 23-36. 
[18] S. Ruan, D. Xiao, Global analysis in a predator-prey system with nonmonotonic functional response, SIAM J. Appl. Math, 61(2001), 1445-1472.

[19] W. Sokol and J.A. Howell , Kineties of phenol oxidation by ashed cell, Biotechnol. Bioeng., 23(1980), 2039-2049.

[20] B. Shulgin, L. Stone and Z. Agur, Pulse vaccination strategy in the SIR epidemic model, Bull. Math. Biol., 60(1998), 1-26.

[21] S.Y. Tang and L. Chen, Density-dependent birth rate, birth pulse and their population dynamic consequences, J. Math. Biol., 44(2002), 185-199.

[22] S. Tang, Y. Xiao, L. Chen and R.A. Cheke, Integrated pest management models and their dynamical behaviour, Bulletin of Math. Biol., 67(2005), 115-135.

[23] W. Wang, H. Wang and Z. Li, The dynamic complexity of a three-species Beddington-type food chain with impulsive control strategy, Chaos, Solitons and Fractals, 32(2007), 1772-1785.

[24] W. Wang, H. Wang and Z. Li, Chaotic behavior of a three-species Beddington-type system with impulsive perturbations, Chaos Solitons and Fractals, 37(2008), 438-443.

[25] R. D. Yang and A. E. Humphrey, Dynamics and steady state studies of phenol biodegeneration in pure and mixed cultures, Biotechnol. Bioeng, 17(1975), 1211-1235.

[26] S. Zhang and L. Chen, Chaos in three species food chain system with impulsive perturbations, Chaos Solitons and Fractals, 24(2005), 73-83.

[27] S. Zhang and L. Chen, A Holling II functional response food chain model with impulsive perturbations, Chaos Solitons and Fractals, 24(2005), 1269-1278.

[28] S. Zhang, F. Wang and L. Chen, A food chain model with impulsive perturbations and Holling IV functional response, Chaos, Solitons and Fractals, 26(2005), 855-866.

[29] S. Zhang, L. Dong and L. Chen, The study of predator-prey system with defensive ability of prey and impulsive perturbations on the predator, Chaos, Solitons and Fractals, 23(2005), 631-643. 